International Journal of Pure and Applied Mathematics

Volume 111 No. 3 2016, 455-465

ISSN: 1311-8080 (printed version); ISSN: 1314-3395 (on-line version)

url: http://www.ijpam.eu

doi: 10.12732/ijpam.v111i3.9

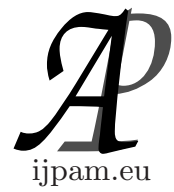

\title{
HIGH-VOLTAGE STARTING SYSTEMS OF COMBUSTION ENGINES
}

\author{
Arkady A. Skvortsov ${ }^{1}$, Vyacheslav P. Khortov ${ }^{2}$ \\ Sergey M. Zuev ${ }^{3}$ \\ ${ }^{1}$ Mechanics of Materials Department \\ Moscow Polytechnic University \\ 107023, Bolshaya Semenovskaya Street 38, \\ Moscow, RUSSIA \\ ${ }^{3}$ Department of Industrial Electronics \\ Moscow Polytechnic University \\ 107023, Bolshaya Semenovskaya Street 38, Moscow, RUSSIA
}

\begin{abstract}
The paper offers analysis of existing starting systems of combustion engines, including ones using low-voltage energy storage units. It also grounds transition of combustion engines' starting systems to higher voltage with use of high-voltage accumulators.
\end{abstract}

AMS Subject Classification: 35QXX, 62P30, 97MXX

Key Words: combustion engines, high-voltage capacitor, differential equations

\section{Introduction}

As it is known, low-voltage capacitor starting systems for combustion engines are still not in mass or even serial production [1]. The matter is for the recent 30 years from the moment of capacitor starting system invention hundreds of tests have been carried out and dozens of related theoretical tasks have been solved [2] and [3]. Results allow making conclusions that can be quite useful in taking decisions linked to industrial use of capacitor starting systems. The main conclusion, however, is that low-voltage systems have no any prospects

\footnotetext{
Received: October 12, 2016

Revised: December 11, 2016

Published: December 17, 2016

${ }^{\S}$ Correspondence author
}

(C) 2016 Academic Publications, Ltd.

url: www.acadpubl.eu 
and the future belongs to high-voltage systems [4] and [5], as far as they are economical, i.e. in comparison to low-voltage systems provide start of combustion engine with significantly lower energy consumption, which implies lower mass of capacitor [6].

\section{Materials and Methods}

Hopelessness of low-voltage systems even with use of molecular and other highvolume types of capacitors can be explained with the following facts. It is known the most important indicator of accumulator batteries applied in combustion engines' starting systems is their internal resistance, which defines maximum power that can be taken from battery [7]. This power is equal to ratio of squared voltage on battery clamps to its quadruplicated internal resistance.

Another one well-known fact is that when reducing environment temperature (and consequently electrolyte) and discharging accumulator, its internal resistance grows [8]. This means the power delivered to network system reduces. At the same time the demands of combustion engine for power in case of temperature reduction on the contrary increases. Therefore, all the attempts of capacitor starting systems' implementation in fact were the attempts to resolve the present difference. At that it was considered accumulator battery would be required only for charging capacitors and, since the internal resistance of the latter ones under otherwise equal conditions was much lower than battery resistance, the power of starting system should significantly increase [9].

It is being typical for pulse capacitors indeed. However low-voltage molecular capacitors, on which subsequently emphasis due to their high storage density had been put, possess approximately the same resistance that is possessed by car accumulators of starting batteries. In principle, the design of molecular capacitor is analogous to accumulator battery, the only difference is that it accumulates energy not due to chemical reaction, but in double electric layer formed by polarized (carbon, for instance) electrode (electronic conductor) and electrolytic solution (ionic conductor) [10]. It is just presence of electrolyte that provides getting of lower internal resistance in molecular capacitor as opposed to accumulator battery [11].

One can assure of the fact due to the following table including values of conductivity for different electrolytic solutions (Table 1).

As far as in battery and molecular capacitors the same electrolyte is applied (acid or alkali), the characteristics of these two accumulators (particularly, in terms of internal resistance) in equivalent to their mass unit appear to be of 
Table 1: Values of conductivity for different electrolytic solutions

\begin{tabular}{|c|c|c|c|c|c|c|c|}
\hline 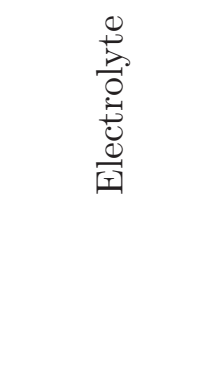 & 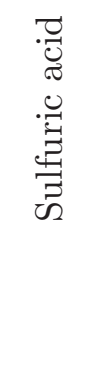 & 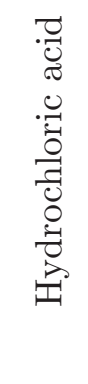 & 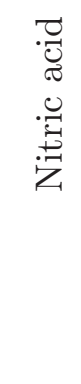 & 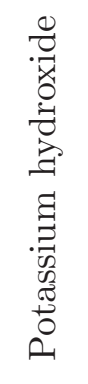 & 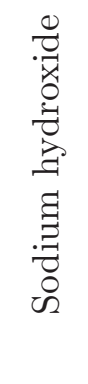 & 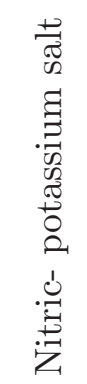 & 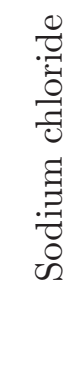 \\
\hline $\begin{array}{l}\text { electrolytic } \\
\text { conductivity, } \\
\mathrm{mS}\end{array}$ & 8,58 & 4,26 & 4,21 & 2,71 & 2,47 & 1,45 & 1,26 \\
\hline
\end{tabular}

the same magnitude.

However, capacitor in a starting system is being intermediate, i.e. additional element in accumulator battery circuit, as well as electric starter. At that the element possesses its own internal resistance.

As a result, it turns out to be that in process of capacitor's chargingdischarging we lose energy of accumulator battery for two times due to the specified internal resistance. This means if there is an accumulator in the starting system the losses of energy in battery increase.

At the present time specialist literature and scientific articles provide two general diagrams for starting system with energy storage units - one implying getting energy directly from the capacitor preliminary charged by accumulator battery and another including parallel working of accumulator battery and capacitor.

When using the first diagram (Figure 1) the volume of capacitor battery is defined by its energy. 


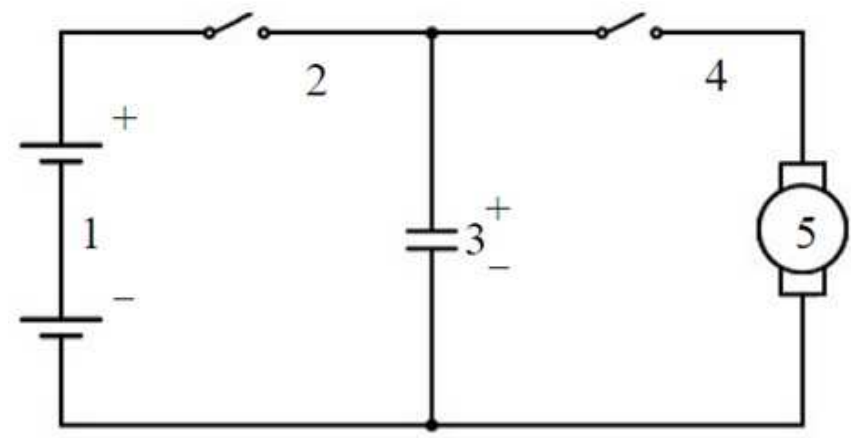

Figure 1: Diagram of starting system with energy capacitors directly from the capacitor preliminary charged by accumulator battery: 1 - car accumulator battery; 2 - charge breaker contacts; 3 - energy capacitor; 4 start switch contacts; 5 - electrical starter.

The amount of energy at the same time should correspond to starter's consumption of definite power multiplied by time of starting $\tau_{0}$. Under low temperatures $\tau_{0}$ significantly increases and can reach $10 \mathrm{sec}$. for gasoline engines and 15 for diesel ones.

Eventually the mass of capacitor approaches the sizes of accumulator battery, while its cost is several times higher - so is there any sense to use such a starting system? It will be more rational for consumers to increase volume of accumulator battery or plant the second one.

In case of diagram using energy capacitor integrated in parallel with accumulator battery (Figure 2) the capacitor takes charge from the battery and subsequently discharges together with it and the starter.

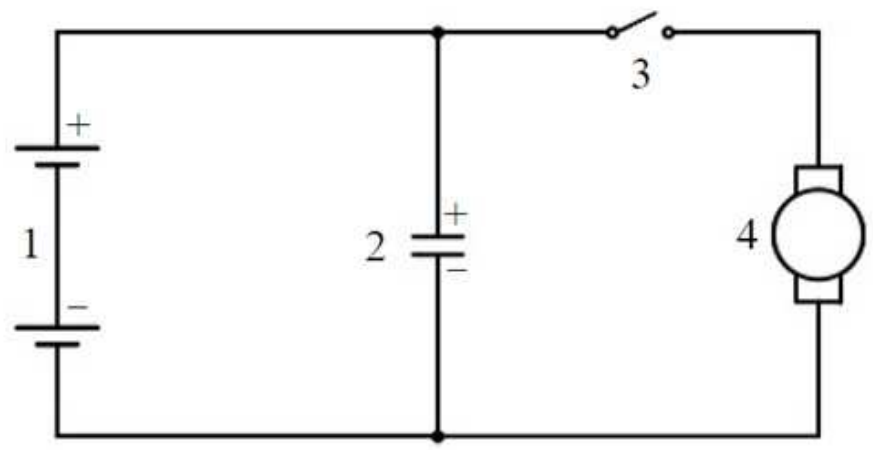

Figure 2: Diagram of starting system with use of energy capacitor integrated to the circuit in parallel with accumulator battery: 1 - car accumulator 
battery; 2 - energy capacitor; 3 - start switch contacts; 4 - electrical starter.

\section{Results and Discussion}

It is well known that energy of capacitor $\mathrm{W}$ is defined by formula 1 :

$$
W=\frac{c u^{2}}{2}
$$

where $c$ - capacitor volume, $u$ - voltage.

This means to charge capacitor to definite level there is a necessity of consuming the same energy for the process of charging. Thus, it takes two times more energy from accumulator.

Indeed, it can be proven in quite a simple way. For example, if we take a charged capacitor $c_{1}$ possessing energy:

$$
W_{1}=\frac{c_{1} u_{1}^{2}}{2}
$$

(where $c_{1}$ - volume of capacitor $1, F ; u_{1}$ - voltage in capacitor $1, V$ ) and connect the same uncharged capacitor to it, in theory we can define energy of each capacitor by reaching achievement equilibrium position, i.e.:

$$
u_{1}=r \cdot i+u_{2}
$$

where $r$ is for electrical resistance of material of plates and capacitor leads, and also taking into account losses in dielectric:

$$
\begin{gathered}
i=-c_{1} \frac{d u_{1}}{d t}=c_{2} \frac{d u_{2}}{d t} \\
u_{2}=u_{1}+c_{1} r \frac{d u_{1}}{d t}
\end{gathered}
$$

On differentiation (5) by time and multiplication by $c_{2}$

$$
c_{2} \frac{d u_{2}}{d t}=c_{2} \frac{d u_{1}}{d t}+c_{1} c_{2} r \frac{d^{2} u_{1}}{d t^{2}}
$$

As a result, we will get differential equation:

$$
\frac{d^{2} u_{1}}{d t^{2}}+\frac{\left(c_{1}+c_{2}\right)}{c_{1} c_{2} r} \frac{d u_{1}}{d t}=0
$$


which can be represented as:

$$
\frac{d^{2} x}{d t^{2}}+a_{1} \frac{d x}{d t}=0
$$

Its solution:

$$
u_{1}=A_{0}+A_{1} e^{-\frac{\mathrm{t}}{\tau}}
$$

where

$$
\begin{gathered}
\frac{1}{\tau}=\frac{c_{1}+c_{2}}{r c_{1} c_{2}} \\
i=-c_{1} \frac{d u_{1}}{d t}=A_{1} \frac{c_{1}}{\tau} e^{-\frac{\mathrm{t}}{\tau}}=A_{1} \frac{c_{1}+c_{2}}{r c_{2}} e^{-\frac{\mathrm{t}}{\tau}}
\end{gathered}
$$

At the beginning of discharging $(t=0) u=u_{1}, u_{2}=0, i=\frac{u_{1}}{r}$. Therefore (9) and (11) lead to

$$
\begin{gathered}
\left.u_{1}\right|_{t=0}=A_{0}+\left.A_{1} i\right|_{t=0}=\frac{u_{1}}{r}=\frac{A_{1}\left(c_{1}+c_{2}\right)}{r c_{2}} \\
A_{1}=\frac{c_{2}}{c_{1}+c_{2}} u_{1} \\
A_{0}=\left.u_{1}\right|_{t=0}-A_{1}=\frac{c_{1}}{c_{1}+c_{2}} u_{1}
\end{gathered}
$$

When the process of charging ends $(t \rightarrow \infty)$

$$
u_{1}=u_{2}=A_{0}
$$

and the energy of capacitors will be equal to:

$$
\begin{gathered}
\frac{c_{1} A_{0}^{2}}{2}=\frac{c_{1}^{3} u_{1}^{2}}{2\left(c_{1}+c_{2}\right)^{2}} \\
\frac{c_{2} A_{0}^{2}}{2}=\frac{c_{1}^{2} c_{2}}{2\left(c_{1}+c_{2}\right)^{2}} u_{1}^{2}
\end{gathered}
$$

In our case $c_{1}=c_{2}$

$$
\frac{c_{1} A_{0}^{2}}{2}=\frac{c_{2} A_{0}^{2}}{2}=\frac{c_{1} u_{1}^{2}}{8}
$$

As a rule, upon consideration the task about charging of capacitor with energy $W_{0}$ from similar (equal electric capacity) charged capacitor we get $\frac{W_{0}}{2}=\frac{c_{1} u_{1}^{2}}{4}$, while with account of losses in dielectric the energy of two capacitors will make $\frac{W_{0}}{4}=\frac{c_{1} u_{1}^{2}}{8}$. 
On the assumption of above-mentioned we can conclude that to charge capacitor to energy $W_{0}$ it should consume $2 W_{0}$, which is not taken into account by engineers of systems that use storage capacitors.

The present conclusion appears, as we can see, from the simplest abstract theorems. However, in practice the situation is much more difficult. Recently developed molecular capacitors with non-aqueous and polymer electrolyte possess even higher internal resistance, than exploited starting batteries do. Therefore, they cannot be applied in car engines' starting systems.

However, there is more to come. Double electrical layer discovered by Helmholtz as early as in 1887 applied in molecular capacitors with acidic and alcaline electrolytes can exist only under voltage not exceeding $0,8 V$ [10]. Consequently, to get the voltage of $12 \mathrm{~V}$ we need at least 15 paralleled elemental capacitors, which not just increases internal resistance of battery, but also provides significant technological difficulties in production process - especially if we take into account that fact that world's motor park in case of transition to capacitor starting systems will require about $190 \mathrm{mln}$. capacitors a year at the price not exceeding the cost of traditional car accumulator battery. What if for cost of molecular capacitor for starting systems - it is even higher.

So, that is how matters of capacitors stand. However, we cannot also disregard the fact the existence of car electrical system with voltage of $12 \mathrm{~V}$ is placed in question too [4] and [5]. For example, in the USA and Japan there are committees dealing with research of possibility of transition of on-board systems to higher voltage. A number of car manufacturers already produce cars with increased voltage in on-board system. However, if we speak about high-voltage starting systems, there is no such agreement of opinion. It seems that with the advent of molecular capacitors it was considered their implementation to starting systems will be quite quick and require no rearrangement of car's on-board system.

Problems linked to low-voltage systems will appearingly make us to refer to high-voltage systems. Since, as our researches revealed, the mass of high-voltage capacitor battery is four time lower, than the mass of low-voltage battery. The reasons for it are the following.

First, high-voltage batteries can use customary electrolytic capacitors represented with one layer structures comprised of aluminium foil (electrodes) and aluminum oxide (dielectric) meant for nominal voltage up to $500 \mathrm{~V}$. This means their internal resistance under otherwise equal conditions can be 500 times lower, than internal resistance of single cell of molecular capacitor with corresponding values of energy and mass. Therefore, the power of such source, if we judge by its direct proportionality to squared voltage and inverse propor- 
tionality to quadruplicate internal resistance, can be very high.

Second, it is well known that KPI of low-voltage electric starter do not exceed $40 \%$. However, in case of transition to higher voltage currents in its plates reduces, as well as losses in them and supply leads, and mass of wires, commutator bars and brushes. The result is the increase of starter KPI, which also has favourable impact on the mass of capacitor battery.

Electrolytic capacitors possess another one feature: the higher their nominal voltage, the higher their energy density is. Thus, there is a practical possibility of realization of starting systems with such capacitors can be performed with minimum voltage of 60-65 $\mathrm{V}$, as far as in case of voltage being lower than $60 \mathrm{~V}$ the mass of battery remains unacceptable. So, the higher the voltage, the lower the mass is. For example, substantiated calculations revealed that in case of voltage of $300 \mathrm{~V}$ the mass of capacitor battery for starting system of modern motor vehicle do not exceed $5 \mathrm{~kg}$. and the prestart charge of such battery can be performed with use of motorcycle battery - we can increase voltage up to, for instance, $300 \mathrm{~V}$ with help of compact and inexpensive transformers, as far as they function for a short moment.

The problem is also facilitated due to the fact that at the present time there are a big number of plants making a specialty out of producing electrolytic capacitors, while the number plants dealing with production of molecular lowvoltage capacitors for combustion engines' starting systems is much lower.

At last, what is for electric starters. There is no necessity of organizing production of high-voltage starters. In principle the problem is solved long ago by other branches of industry. Thus, the industry of manual electric hand tools today produces high-voltage electrical engines at rates exceeding demands of automobile manufacturing for several times. If we, in addition, take into account the fact that electric starter of future will be presented with highvoltage electrical machine possessing permanent-magnet excitation and built-in reduction unit, its overall dimensions and cost will be patently more effective in comparison to low-voltage electric motors.

We should also notice the starting system of high-voltage capacitor confuses many consumers and manufacturers with just presence of high voltage. But there is no need to worry - since in the earliest days of transport facilities' development the on-board voltage made just $6 \mathrm{~V}$. However, in connection with increase of amount of consumers and power branches related to water transport and aviation have transited to high voltage and alterating current long ago, while low voltage has remained a supportive factor. And only cars' on-board systems still use low voltage of 12 or $24 \mathrm{~V}$ (in case of starting systems of multicylinder combustion engines. There are many reasons for such a situation - for 
example, concerns linked to electrical safety. However, all of them don't stand up to scrutiny just due to the fact the on-board voltage of manufactured today automobiles makes 110-400 $\mathrm{V}$. So the problem of electrical safety possesses many developed solutions.

In general, whether we want or not, transition of cars' on-board systems to increased voltage is inevitable. First, the number of electrical energy consumers in modern automobile just constantly grows. Second, the power of energy consumers also increases (according to data of American experts, by 2020 it will make about 5-7 kW). Third, achievements in the development of semiconducting converter equipment provides easy transition to any voltage being optimal for this or that engine electrical system (for example, in case of transition to increased voltage all the low-voltage consumers - sensors, indication devices, etc. - can be supported by low voltage). Fourth, gas-discharge and photodiode lighting equipment in automobile provides opportunity of several times increase of reliability of car's electrical lamps and luminous efficiency with decrease of mass. At that gas-discharge lamps require voltage of no less, than $80 \mathrm{~V}$. Fifth, implementation of high-voltage capacitor starting systems allows us to solve the main problem in transition to higher voltage - the accumulator battery possessing increased voltage on its terminals. The simplest diagram of highvoltage system is shown in Figure 3. It is similar to traditional system, except for it possesses high-voltage starter and voltage converter represented in Figure 4. Design of voltage converter is also quite simple: 2-4 transistors working as the key at high frequency (up to $40 \mathrm{kHz}$ ), which provides for its dimension less than $200 \mathrm{~g}$.

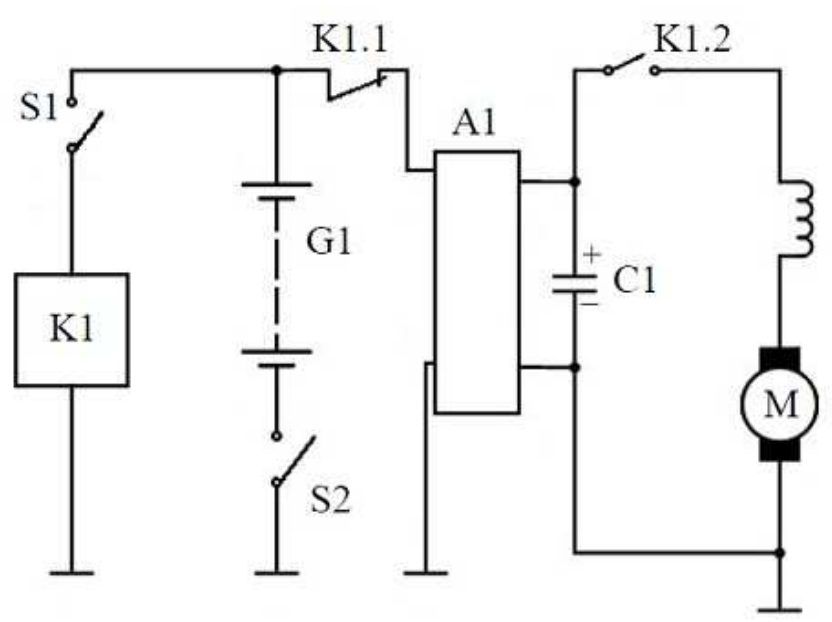

Figure 3: Schematic electrical diagram of starting system: $K 1$ - the coil of 
the traction relay; $S 1$ - key ignition switch; $G 1$ - accumulator battery; $S 2$ capacitor battery switch; $A 1$ - voltage converter; $C 1$ - capacitive storage; $K 1.1, K 1.2$ - start switch contacts; $M$ - electric starter.

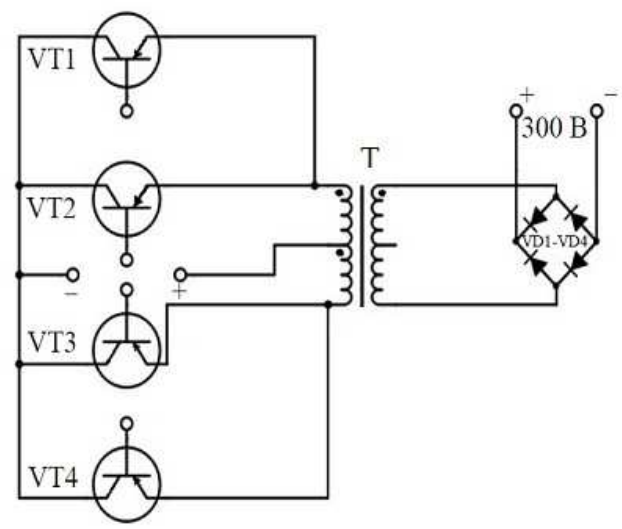

Figure 4. Schematic electrical diagram of voltage converter: VT1 . VT4 transistors, $V D 1 \ldots V D 4$ - diode bridge; $T P$ - ferritic output transformer.

\section{Conclusion}

Surely, the transition of inboard voltage to higher values is quite a difficult task. However, the fact that it is dictated by the present time remains evident. And we should start this transition just with high-voltage starting systems.

\section{Acknowledgements}

The present work is made with financial support from Ministry of Education and Science of the Russian Federation within the framework of Government decree No. 218 (contract No. 02.G25.31.0138 of 12.01.2015).

\section{References}

[1] G. Grzeczka, P. Swoboda, Analysis of the possibility of use lithium: Ion as a starting battery on the ship engine room, Solid State Phenomena, 236 (2015), 106-112, doi: 10.4028/www.scientific.net/SSP.236.106. 
[2] M.-Y. Lee, D.H. Lim, S.C. Kim, Evaluation of the effect of operating parameters on thermal performance of an integrated starter generator in hybrid electric vehicles, Energies, 8 (2015), 8990-9008, doi: 10.3390/en8088990.

[3] S.K. Gurusamy, C. Rajagopalan, R. Sateesh Kumar, B. Ashok, Reducing starting current for existing commercial vehicle engines, SAE Technical Papers, January 2015, 14th Symposium on International Automotive Technology, SIAT 2015, Code 110846, doi: 10.4271/2015-26-0042.

[4] A. Weber, 48-volt systems will help reduce wiring costs. Assembly, 58 (2015).

[5] D. Stojan, P. Sever, J. Horvat, High voltage converter design for a belt alternator starter, Przeglad Elektrotechniczny, 87 (2011), 179-182, doi: 10.1109/IECON.2008.4757955.

[6] S.T. Chen, B. Lequesne, R.R. Henry, Y.H. Xue, J.J. Ronning, Design and testing of a belt-driven induction starter-generator, IEEE Transactions on Industry Applications, $\mathbf{3 8}$ (2002), 1525-1533, doi: 10.1109/TIA.2002.805563.

[7] M.A. Laughton, D.F. Warne, Electrical Engineer's Reference Book: Sixteenth Edition, Elsevier (2003).

[8] J. Liu, T. Ning, S. Cao, F. Zhang, Study of the application of super-capacitors in diesel cold start, Lecture Notes in Electrical Engineering, 29 (2014), 691-697, doi: 10.1007/9783-642-54233-6_76.

[9] Z. Cui, R. Wang, The modeling and simulation of super-capacitor in diesel engine starting process. Applied Mechanics and Materials, 229-231 (2012), 1967-1970, doi: 10.4028/www.scientific.net/AMM.229-231.1967.

[10] C. Pean, B. Rotenberg, P. Simon, M. Salanne, Understanding the different (dis)charging steps of supercapacitors: Influence of potential and salvation, Electrochimica Acta, 206 (2016), 504-512, doi: 10.1016/j.electacta.2016.02.106.

[11] P. Ray, S. Dohm, T. Husch, C. Schtter, K.A. Persson, A. Balducci, B. Kirchner, M. Korth, Insights into Bulk Electrolyte Effects on the Operative Voltage of Electrochemical Double-Layer Capacitors, Journal of Physical Chemistry C, 120 (2016), 12325-12336, doi: 10.1021/acs.jpcc.6b00891. 
